\title{
Endovascular Embolization of Brain Arteriovenous Malformations with Eudragit-E
}

\author{
Goichiro TAmura, ${ }^{1}$ Noriyuki KATO, ${ }^{1}$ Tomosato YAMAZAKI, ${ }^{1}$ Yoshimitsu AKUTSU, ${ }^{1}$ \\ Hisayuki HosOO, ${ }^{1}$ Hiromichi KASUYA, ${ }^{1}$ and Makoto SONOBE ${ }^{1}$
}

\author{
${ }^{1}$ Department of Neurosurgery, Mito Medical Center, National Health Organization, \\ Higashi Ibaraki, Ibaraki
}

\begin{abstract}
Eudragit-E was originally developed as a non-adhesive liquid embolic material in the late 1990 s and is a copolymer of methyl and butyl methacrylate and dimethylaminoethyl methacrylate that is dissolved in ethanol and iopamidol. This material has been used for endovascular embolization of brain arteriovenous malformations (AVMs) for some time but is currently not widely used. Because safety and feasibility of Eudragit-E has not been well documented, we here report our experience using this material for treating 22 human brain AVMs. From June 1998 to February 2014, 30 endovascular procedures using Eudragit-E were performed to treat 22 patients, including 14 men and 8 women with a mean age of 41.1 years (15-70 years). The mean follow-up period was 56 months (12-129 months), and the Spetzler-Martin grades were I (4 patients), II (9 patients), III (5 patients), and IV (4 patients). Residual AVMs were treated with stereotactic radiosurgery or surgery. The rate of complete obliteration with embolization alone was $27.3 \%$. The overall obliteration rate after endovascular embolization with/without subsequent stereotactic radiosurgery or surgery was $\mathbf{7 2 . 7 \%}$. Eudragit-E caused two cases of cerebral infarction. One case of intracerebral hemorrhage due to postoperative hemodynamic changes also occurred. The rate of complications directly related to embolization was $\mathbf{1 0 . 0} \%$. The safety and effectiveness of Eudragit-E embolization were satisfactory.
\end{abstract}

Key words: Eudragit, arteriovenous malformation, embolization, liquid embolic material

\section{Introduction}

Treatment of brain arteriovenous malformations (AVMs) generally requires multimodal approaches involving endovascular embolization, surgical excision, and stereotactic radiosurgery. Historically, endovascular embolization of brain AVMs has been achieved using various types of embolic materials. ${ }^{1-3)}$ The most widely used liquid embolic materials today are n-butyl 2-cyanoacrylate (NBCA, Histoacryl; Braun, Melsungen, Germany) and Onyx (Covidien/ev3, Irvine, California, USA).

Eudragit-E (Eudragit-E 100; Evonik Rohm GmbH, Darmstadt, Germany) was originally developed by Yamashita et al. in 1996, ${ }^{4)}$ but has not been widely used for endovascular embolization of brain AVMs. It was originally approved by the United States Food and Drug Administration in 1968, and has been used for many years as a coating material for orally administered tablets. ${ }^{5,6)}$ The Eudragit-E

Received September 3, 2014; Accepted December 1, 2014 mixture consists of a cationic copolymer of methyl methacrylate, butyl methacrylate, and dimethylaminoethyl methacrylate in a ratio of 1:1:2 that is dissolved in $50 \%$ ethanol and $50 \%$ iopamidol. Yamashita et al. reported the first successful embolization of canine and rat renal arteries using this polymer. ${ }^{4)}$ Hyodo et al. have reported their experience with endovascular embolization of brain AVMs since 1983 using different embolic materials, one of which was Eudragit-E. ${ }^{3)}$ Oishi et al. reported a case of scalp AVM that was successfully embolized with Eudragit-E before surgical removal. ${ }^{7)}$ Although Eudragit-E has been used for a long time in our hospital and some institutions in Japan, few reports have been published in which the safety and feasibility of Eudragit-E for treating human brain AVMs were assessed. With the approval of our Institutional Review Board and informed consent from patients, we have been using Eudragit-E for more than 15 years to endovascularly treat brain AVMs. Herein we report our experience treating 22 human brain AVMs with Eudragit-E. 


\section{Materials and Methods}

\section{Patients}

We retrospectively reviewed our patient database. From June 1998 to February 2014, 37 patients with brain AVMs underwent endovascular embolization in our hospital. Of these 37 cases, patients who were followed up for less than 1 year after the initial embolization and those who were treated with other embolic materials were excluded from this study. Eudragit-E was used in 22 patients in a total of 30 endovascular procedures. The mean follow-up period was 56 months (range, 12-129 months).

Eudragit-E was used as the primary embolic material in our hospital. Onyx was approved in 2009 in Japan as the first liquid embolic material for brain AVMs, but Onyx embolization was originally designed as pretreatment for subsequent surgical removal. Because our treatment strategy included subsequent gamma-knife radiosurgery following embolization, patients were similarly treated with Eudragit-E after 2009 with the approval of our Institutional Review Board and informed consent from patients and their family. Seven new patients have been treated with Eudragit-E since 2009.

\section{Preparation of Eudragit-E}

Eudragit-E is supplied as off-white granules. Preparation of $10 \%$ Eudragit-E mixture was performed in our pharmacy department as previously described. $\left.{ }^{4}\right)$ Briefly, $4 \mathrm{~g}$ solid Eudragit-E was dissolved in 20 $\mathrm{ml}$ absolute ethanol and stirred vigorously for 1 hour. Twenty milliliters iopamidol $300 \mathrm{mgI} / \mathrm{ml}$ was added to the solution and stirred immediately at room temperature for several hours to produce a transparent $10 \%$ Eudragit-E mixture. The mixture was filtered through a sterile microfilter with a pore diameter of $0.22 \mu \mathrm{m}$ and divided into $2-\mathrm{ml}$ vials. The vials were crimped to avoid ethanol evaporation and autoclaved at $115^{\circ} \mathrm{C}$ and $3 \mathrm{~atm}$ for 30 minutes.

\section{Treatment strategy}

Endovascular treatment is considered to be the first treatment option in our hospital unless decompressive craniotomy and hematoma removal are urgently required. If complete obliteration could not be achieved with one or a few sessions of endovascular embolization, most patients were subsequently treated with gamma knife radiosurgery (Leksell Gamma Knife Unit; Elekta, Norcross, Georgia, USA). Staged gamma knife radiosurgery was performed to treat large AVMs as described previously. ${ }^{8)}$ AVMs located in superficial cortical areas were surgically removed after endovascular embolization, depending on patient preferences. After gamma knife radiosurgery, follow-up magnetic resonance imaging (MRI) and computed tomography (CT) images were evaluated every 6 months for 3 years. Digital subtraction angiography (DSA) was performed at 1 year and 3 years after the initial embolization. Once complete obliteration of the AVM was confirmed, patients were followed up annually with MRI and CT.

\section{Endovascular procedure}

Endovascular embolization was performed under general anesthesia using a standard biplane angiographic system (Philips Allura Xper FD; Philips Healthcare, Andover, Massachusetts, USA). A 7 Fr-long sheath was placed in the right femoral artery, and a 7-Fr guiding catheter was inserted into the internal carotid or vertebral artery using standard coaxial techniques. The guiding catheter was continuously flushed with heparinized saline. DSA was performed to analyze the meticulous angioarchitecture of the nidus, feeding arteries, and drainage veins. The tip of the microcatheter was navigated through the feeding artery as close as possible to the nidus. We usually use Marathon (Covidien/ev3), Balt Magic B-115H-ST (Balt, Montmorency, France), or Excelsior SL-10 (Stryker, Fremont, California, USA) microcatheters, but all types of microcatheters are compatible with Eudragit-E. For cases with high-flow shunting, the flow was reduced by placing a balloon catheter at the proximal position of the feeding artery. In most cases, the Hyperballoon series (HyperForm or HyperGlide; Covidien/ev3) or Scepter microballoons (Scepter C or Scepter XC; Microvention, Tustin, Californai, USA) were used as the balloon catheter. The dead space volumes of Marathon, Balt Magic B-115H-ST, and Excelsior SL-10 are $0.23 \mathrm{ml}, 0.34 \mathrm{ml}$, and $0.29 \mathrm{ml}$, respectively. The microcatheter lumen was flushed with $0.3 \mathrm{ml}$ to $0.4 \mathrm{ml} 70 \%$ ethanol to prevent undesired precipitation of Eudragit-E prior to its injection. Vasospasm was rarely observed upon injection of this amount of ethanol. Eudragit-E was loaded into a 1-ml syringe, and approximately $0.05 \mathrm{ml}$ to $0.1 \mathrm{ml}$ was slowly and repeatedly injected into the nidus under roadmap guidance. Due to the non-adhesive nature of Eudragit-E, catheter trapping on the cast never occurred regardless of the amount of time required to perform the embolization procedure. We were able to stop and restart injection of Eudragit-E at any time without pulling off the microcatheter. For example, after a few sessions of repeated EudragitE injection, we could examine the residual nidus by injecting contrast medium from the guiding 
catheter without removing the microcatheter tip from the pre-formed cast. Eudragit-E injection was continued until it back-flowed into the feeding artery. The microcatheter was aspirated with a 5-ml syringe to prevent distal migration of Eudragit-E fragments and then removed by careful traction. The same embolization procedure was used for other feeding arteries.

\section{Results}

\section{Case 1}

A 50-year-old male presented to the emergency department with an intraventricular hemorrhage due to a ruptured AVM (Spetzler-Martin grade III). MRI and DSA revealed that the AVM was located in the right corpus callosum (Fig. 1A). The nidus was approximately $4 \mathrm{~cm}$ in diameter, with feeding arteries from both anterior cerebral arteries and right posterior cerebral artery. The AVM drained into the right inferior cerebral vein and straight sinus. Endovascular embolization was performed using a proximal flow control technique. Eudragit-E was injected into the nidus and was clearly visualized with fluoroscopy (Fig. 1B). No complications occurred during the procedure. Only the anterior half of the AVM was embolized after the first embolization procedure. Postoperative CT and MRI showed the Eudragit-E cast and residual AVM (Fig. 1C, D). The artifact caused by the Eudragit-E cast on CT was minimal, and we could easily distinguish the cast from the residual nidus on $\mathrm{T}_{2}$-weighted MRI. The patient underwent a second embolization and gamma knife radiosurgery. Postoperative DSA (Fig. 1E) and MRI revealed complete obliteration of the AVM.

\section{Case 2}

A 16-year-old female was admitted to our emergency department with an intracerebral hemorrhage in the left parietal lobe. DSA confirmed the SpetzlerMartin grade of II. The nidus was approximately $17 \mathrm{~mm}$ in diameter with feeders from the left angular artery and drainers into cortical veins (Fig. 2A). Endovascular embolization using Eudragit-E was performed without complications (Fig. 2B). No residual AVM was observed with DSA at the end of the endovascular procedure (Fig. 2C). However, follow-up MRI and DSA performed approximately 6 months later revealed recurrence of the AVM (Fig. 2D). The lesion was located on the cerebral surface, and the patient and her family preferred surgical excision, which was performed without complications. Intraoperative findings showed that the vessels and nidus that had been embolized with Eudragit$\mathrm{E}$ were elastic and whitish in color (Fig. 2E). The embolized vessels were clearly distinguishable from the non-embolized vessels and were easily handled without tearing the vessel. Pre-surgical embolization with Eudragit-E made surgical excision easier.

\section{Characteristics of patients and AVMs}

Of the 22 patients, there were 14 (63.6\%) men and eight $(36.4 \%)$ women with a mean age of 41.1 years (range, 15-70 years). Initial clinical presentation included 11 cases of intracranial hemorrhage $(50 \%), 3$ cases of seizures without hemorrhage
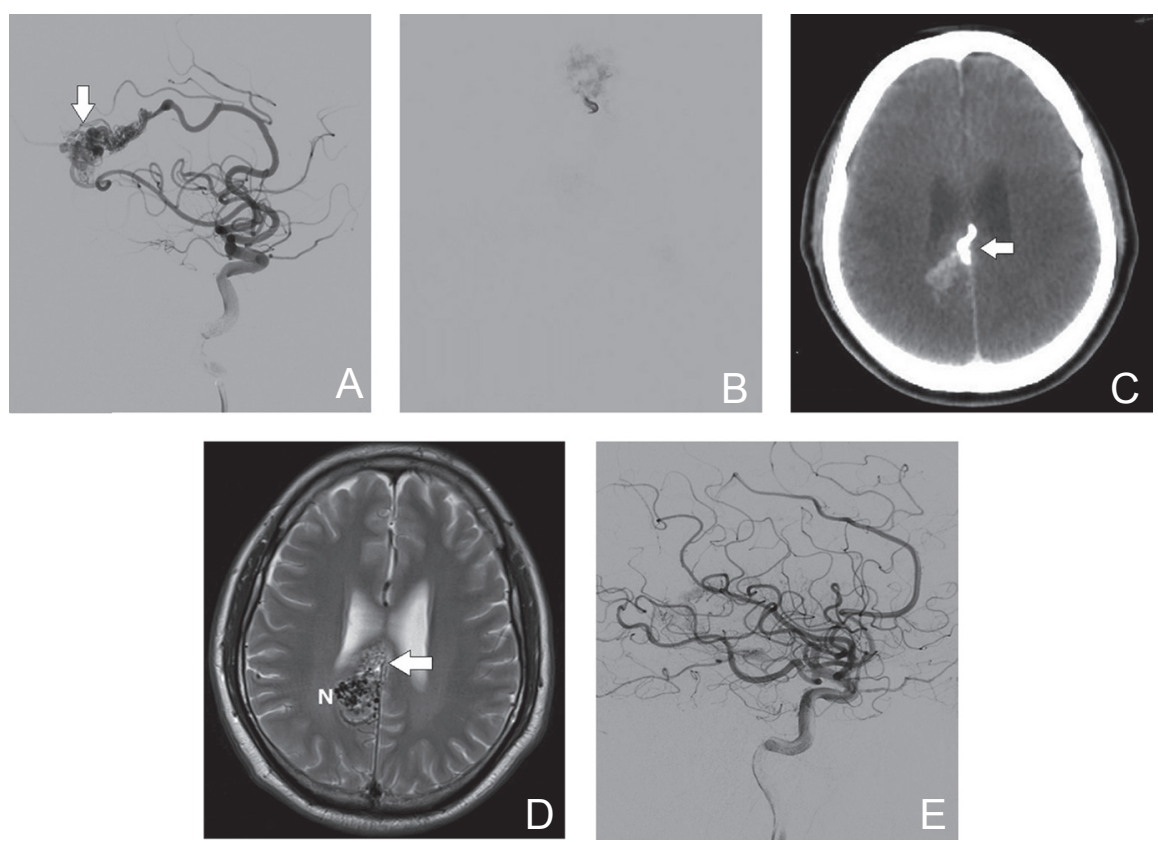

Fig. 1 A 50-year-old male with a ruptured AVM (Spetzler-Martin grade III). A: DSA (lateral view, right internal carotid angiography) shows the nidus (arrow) and main feeding artery from the right anterior cerebral artery. B: Injection of Eudragit-E is clearly visible with fluoroscopy. C: Postoperative CT image (axial view) shows the cast of EudragitE (arrow) with minimal artifacts. D: Postoperative $\mathrm{T}_{2}$-weighted MRI (axial view) reveals the Eudragit-E cast (arrow) and residual nidus $(\mathrm{N})$. E: Postoperative DSA image (lateral view) after two sessions of endovascular embolization and gamma knife radiosurgery shows no residual AVM. AVM: arteriovenous malformation, CT: computed tomography, DSA: digital subtraction angiography, MRI: magnetic resonance imaging. 

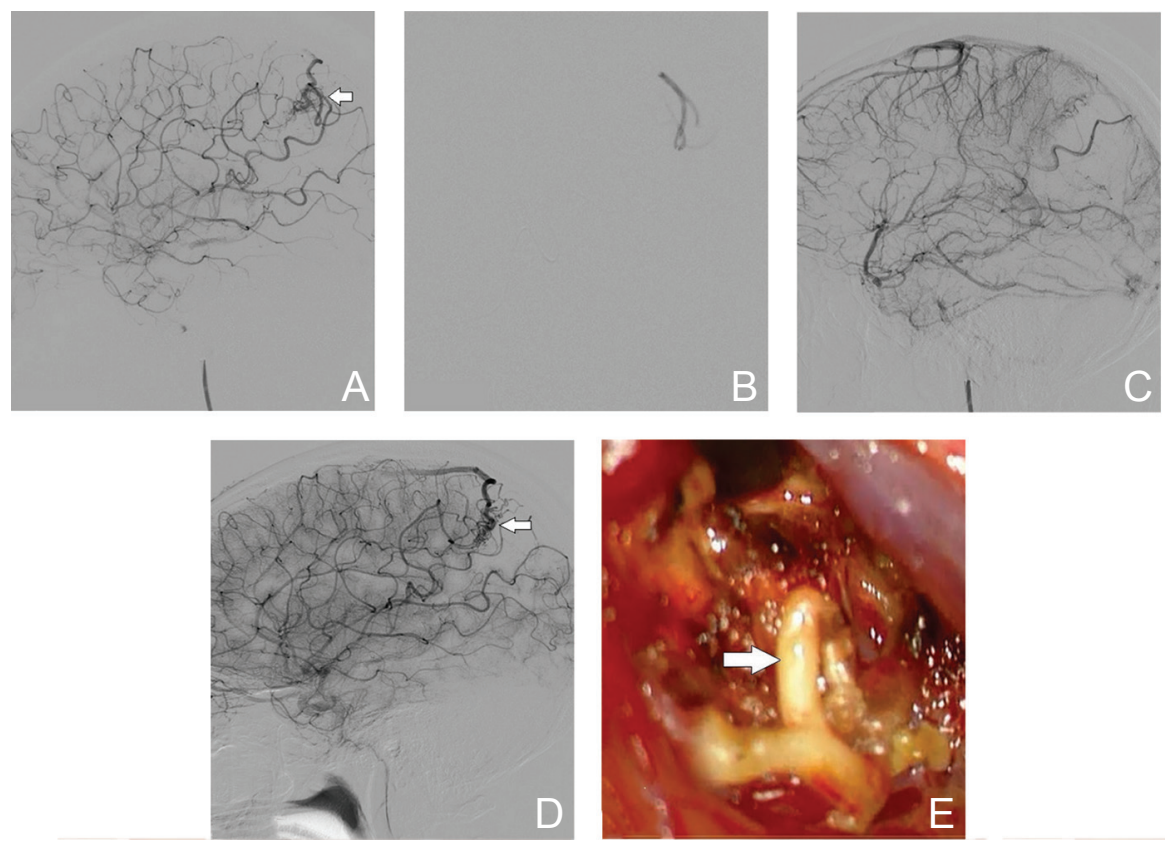

Fig. 2 A 16-year-old female with an intracerebral hemorrhage from a ruptured AVM (Spetzler-Martin grade II). A: DSA (lateral view, left internal carotid angiography) reveals the nidus (arrow) with feeders from the left angular artery. B: Injection of Eudragit-E is clearly visible with fluoroscopy. C: Postoperative DSA (lateral view) shows no residual AVM. D: Follow-up DSA performed 6 months later reveals the recurrence of AVM (arrow). E: Blood vessels that were embolized with Eudragit-E (arrow) are elastic and whitish in color. AVM: arteriovenous malformation, CT: computed tomography, DSA: digital subtraction angiography.

Table 1 The rates of complete obliteration using Eudragit-E (number of cases)

\begin{tabular}{lccccccc}
\hline Spetzler-Martin grade & I & II & III & VI & I-II & III-IV & All \\
\hline Complete obliteration & $50.0 \%$ & $33.3 \%$ & $0 \%$ & $25 \%$ & $38.5 \%$ & $11.1 \%$ & $27.3 \%$ \\
after embolization & $(2 / 4)$ & $(3 / 9)$ & $(0 / 5)$ & $(1 / 4)$ & $(5 / 13)$ & $(1 / 9)$ & $(6 / 22)$ \\
Complete obliteration & $100.0 \%$ & $88.9 \%$ & $60.0 \%$ & $25.0 \%$ & $92.3 \%$ & $44.4 \%$ & $72.7 \%$ \\
after all treatments & $(4 / 4)$ & $(8 / 9)$ & $(3 / 5)$ & $(1 / 4)$ & $(12 / 13)$ & $(4 / 9)$ & $(16 / 22)$ \\
\hline
\end{tabular}

(13.6\%), 3 cases of neurological deficits (13.6\%), 3 cases of headaches $(13.6 \%)$, and 2 incidental findings $(9.1 \%)$.

The size, location, and angiocharacteristics of AVMs were evaluated with MRI and DSA. All AVMs were classified according to the SpetzlerMartin classification (grade I in 4 patients, II in 9 patients, III in 5 patients, and IV in 4 patients). Our patient group included one infratentorial and 21 supratentorial lesions (4 frontal, 5 parietal, 8 temporal, 2 occipital lobes, 1 cerebellum, 1 corpus callosum, and 1 basal ganglia). The AVM nidus diameter was less than $3 \mathrm{~cm}$ in 14 patients (63.6\%) and more than $3 \mathrm{~cm}$ in 8 patients $(36.4 \%)$.

\section{Obliteration rate}

We performed a total of 30 embolization procedures on 22 patients. Eight patients underwent two embolization procedures. Eudragit-E alone was used in 28 procedures, whereas Eudragit-E plus coils were used in two procedures to obliterate associated aneurysms. Nine patients were treated with endovascular embolization alone. Twelve patients were treated with embolization plus subsequent gamma knife radiosurgery. One patient was treated with embolization followed by planned surgical excision. The rate of complete obliteration achieved with our endovascular treatment alone was $27.3 \%(6 / 22)$, whereas the overall complete obliteration rate after embolization with/without additional treatments was $72.7 \%(16 / 22)$. The results are summarized in Table 1.

\section{Complication rate}

A total of seven adverse effects (two cerebral infarctions and five intracranial hemorrhages) occurred that caused permanent neurological deficits; three of them $(10.0 \%, 3 / 30)$ were directly related to the embolization procedure. Two cases of cerebral infarction were caused by undesired obliteration of normal vessels. One intracerebral hemorrhage occurred on the day after the endovascular treatment and was suspected to be due to hemodynamic changes. Trapping of microcatheters with EudragitE was not observed in any patients in our study.

Three additional cases of intracranial hemorrhage occurred during the latency period after gamma knife radiosurgery, at 4 months, 17 months, and 35 months after the last radiation. The first one (Spetzler-Martin grade III) was fatal, and the mortality 
Table 2 The rates of permanent complications using Eudragit-E (number of procedures or cases)

\begin{tabular}{|c|c|c|c|c|c|c|c|}
\hline Spetzler-Martin grade & I & II & III & IV & I-II & III-IV & All \\
\hline $\begin{array}{l}\text { Complications after } \\
\text { embolization procedures }\end{array}$ & $\begin{array}{c}0 \% \\
(0 / 4)\end{array}$ & $\begin{array}{c}9.1 \% \\
(1 / 11)\end{array}$ & $\begin{array}{c}12.5 \% \\
(1 / 8)\end{array}$ & $\begin{array}{c}14.3 \% \\
(1 / 7)\end{array}$ & $\begin{array}{l}6.7 \% \\
(1 / 15)\end{array}$ & $\begin{array}{l}13.3 \% \\
(2 / 15)\end{array}$ & $\begin{array}{l}10.0 \% \\
(3 / 30)\end{array}$ \\
\hline $\begin{array}{l}\text { Complications after all } \\
\text { treatments }\end{array}$ & $\begin{array}{c}25.0 \% \\
(1 / 4)\end{array}$ & $\begin{array}{c}33.3 \% \\
(3 / 9)\end{array}$ & $\begin{array}{c}40.0 \% \\
(2 / 5)\end{array}$ & $\begin{array}{c}25.0 \% \\
(1 / 4)\end{array}$ & $\begin{array}{l}30.8 \% \\
(4 / 13)\end{array}$ & $\begin{array}{c}33.3 \% \\
(3 / 9)\end{array}$ & $\begin{array}{l}31.8 \% \\
(7 / 22)\end{array}$ \\
\hline
\end{tabular}

rate in this study was therefore $4.5 \%(1 / 22)$. The diameter of the nidus was more than $5 \mathrm{~cm}$, and only its meningeal feeder was embolized at the time of endovascular treatment. Two-stage gamma knife radiosurgery was subsequently performed, but intracerebral hemorrhage occurred 4 months after the second radiosurgery. Emergency decompressive craniotomy with hematoma removal was unsuccessful for rescuing the patient. Another case of intracerebral hemorrhage occurred 27 months after embolization of the AVM. The residual AVM was surgically excised at the time of decompressive craniotomy. The overall complication rate was therefore $31.8 \%(7 / 22)$. The rate of complications directly related to embolization was $10.0 \%(3 / 30)$. The results are summarized in Table 2.

\section{Discussion}

NBCA and Onyx are the most widely used liquid embolic materials for AVM embolization today. NBCA is especially suitable for AVMs with highflow shunts, but NBCA is difficult to handle even by experienced neuro-interventionists because it polymerizes very quickly. Onyx, on the other hand, solidifies more slowly, allowing prolonged and controlled injection. Onyx consists of an ethylene vinyl alcohol copolymer dissolved in dimethyl sulfoxide (DMSO) with tantalum powder added for radiopacity.

Eudragit-E is a cationic copolymer that attracts negatively charged blood elements and precipitates immediately upon injection into blood. Eudragit-E is a non-adhesive agent that is similar to Onyx and that has little risk of catheter adhesion compared to adhesive agents such as NBCA. Eudragit-E is even less adhesive than Onyx, and we did not experience any catheter adhesion in this study. Slow, repeated injection was easily achieved due to the cohesive but non-adhesive nature of Eudragit-E. Withdrawal of the microcatheter tip from the Eudragit cast was not required, for example, when we examine the residual nidus with contrast injection from the guiding catheter. Catheter trapping on the cast never happened in this study.

Eudragit-E is very easy to handle. Onyx requires the "plug and push" technique for effective embolization. To produce effective plug and also to avoid catheter trapping, Onyx may require a straight vessel of some length between the microcatheter tip and the nidus. On the other hand, Eudragit-E does not require the formation of plug and can be injected by simple "pushing." This allows Eudragit-E to be injected into feeders and nidus of almost any angiostructure. Furthermore, Onyx requires only DMSOcompatible microcatheters, while Eudragit-E shows no incompatibility with any type of microcatheter. ${ }^{9)}$ Ease of use and compatibility with any type of microcatheter are great advantages of Eudragit-E, which is especially beneficial when approaching AVMs with complex angiostructures.

Eudragit-E is not inherently radiopaque, but the Eudragit-E mixture can be clearly visualized with fluoroscopy due to the contrast medium, iopamidol (Figs. 1B, 2B). A former criticism of Eudragit-E was that it might be less visible on a biplane angiographic system, but recent advancements in radiological devices have allowed clear visualization. Reduction of the flow into the nidus by proximal balloon placement is also effective to visualize the Eudragit-E during embolization (Fig. 1B).

Iopamidol, however, gradually diffuses out of the precipitated cast. Thus, the radiopacity of the cast will decay with follow-up DSA or CT images. This characteristic of reduced radiopacity is especially advantageous for subsequent stereotactic radiosurgery. Tantalum powder added to Onyx for radiopacity makes it difficult to plan subsequent radiosurgery or repeated endovascular embolization. Tantalum produces significant artifacts on postembolization CT. ${ }^{10)}$ Onyx casts appear hypointense on $\mathrm{T}_{2}$-weighted MRI, similar to untreated AVMs that appear hypointense due to their flow voids. These characteristics of Onyx make it difficult to discriminate the residual targets and to apply appropriate doses during subsequent stereotactic radiosurgery. ${ }^{10,11)}$ Eudragit-E, however, produces little artifact (Fig. 1C, D), allowing more accurate planning of subsequent gamma knife radiosurgery. Eudragit-E embolization is therefore especially advantageous as pretreatment for stereotactic radiosurgery. This may be one reason that our obliteration rate $(66.7 \%, 8 / 12)$ after 
embolization plus radiosurgery was satisfactory.

Presurgical embolization using Eudragit-E rendered AVMs easy to treat during subsequent surgical excision, as seen in Case 2. Blood vessels compacted with Onyx are reported to be elastic, bluish in color, and easy to hold or pull with forceps without tearing blood vessels. ${ }^{12,13)}$ Eudragit-E also renders the blood vessels firm and easy to handle, although the vessels appear whitish in color (Fig. 2E). Therefore, Eudragit-E and Onyx are similarly effective for presurgical embolization.

One disadvantage of Eudragit-E is that it is easily fragmented, especially when the blood flow is rapid. To avoid distal migration of Eudragit-E or venous occlusion, we utilize the proximal flow control technique for cases with high-flow shunting such as that in Case 1. Placing a balloon catheter proximal to the target feeders as close as possible to the nidus is effective for controlling the flow rate and preventing fragmentation of Eudragit-E.

In our series, the overall obliteration rate after all treatments was $72.7 \%$ (Table 1). Complete obliteration was more frequently achieved with lower grade AVMs (grade I: $100 \%$, grade II: $88.9 \%$, grade III: $60.0 \%$, grade IV: $25.0 \%$, grade I-II: $92.3 \%$, grade III-IV: $44.4 \%$ ). Pierot et al. reported a similar result after Onyx embolization with subsequent radiosurgery: complete obliteration was achieved in $71.4 \%$ of grade I-II AVMs and 50\% in grade III-IV AVMs. ${ }^{14)}$ Zabel du Bois et al. also reported a similar result after Onyx embolization and radiosurgery: 90\% in grade I-II AVMs and 59\% in grade III-IV AVMs. ${ }^{15}$ The obliteration rate achieved with embolization alone was $27.3 \%$ in our study. The obliteration rate of Onyx embolization alone is reported to be $16-54 \%,{ }^{16-18)}$ whereas that of NBCA is $6-33 \%{ }^{19,20)}$ (Table 3). These results indicate that the efficacy of Eudragit-E as a liquid embolic material for brain AVMs is not inferior to Onyx or NBCA.

The rate of embolization-related complications that caused permanent neurological deficits in our study was $10.0 \%$ (Table 2). Previous reports indicate that the complication rate of Onyx embolization alone is $5-8 \%,{ }^{17,18)}$ and that of NBCA is $2-7 \% .^{19,20)}$ Thus, the complication rate of Eudragit-E embolization may be similar to or slightly higher than that of Onyx or NBCA. This may be partly due to its tendency to fragment easily. The proximal flow control technique described above may be effective for reducing embolization-related complications in future treatments. The complication rate after Onyx or NBCA embolization followed by stereotactic radiosurgery ranges from $5 \%$ to $50 \% .{ }^{13,14,21,22)}$ Our overall complication rate after all treatments was $31.8 \%$ (Table 2). Therefore, our combination therapy using Eudragit-E embolization and subsequent radiosurgery or surgery did not necessarily cause more complications than other previously reported multimodal treatments.

Eudragit-E is dissolved in 50\% ethanol, which may be a concern for possible inflammatory reactions to the vessels. Yamashita et al. reported their histological studies of cane renal arteries after injection of Eudragit-E. ${ }^{4)}$ No acute inflammatory reaction took place within 1 week, though mild chronic reactions were observed a few weeks later. Sampei et al. compared histological reactions of rodent cerebral vessels to DMSO and $40 \%$ ethanol and showed $40 \%$ ethanol was safer when injected at a rate of $0.04 \mathrm{ml} / \mathrm{s}^{23)}$ Arakawa et al. injected $50 \%$ ethanol containing Eudragit-E into swine blood vessels at 0.02 $\mathrm{ml} / \mathrm{s}$ and did not experience any adverse effects. ${ }^{9)}$ These reports suggest that ethanol may not cause severe inflammation as long as a small amount is injected at a slow rate.

Arakawa et al. proposed that inflammatory responses may be caused by the Eudragit polymer rather than the ethanol solvent. ${ }^{9)}$ They showed that the ethanol/ iopamidol mixture did not cause any endothelial damage and inflammation might rather be caused by

Table 3 The rates of complete obliteration and permanent complications using different liquid embolic materials

\begin{tabular}{lcc}
\hline Materials, authors, year & Rate of complete obliteration & Rate of permanent complications \\
\hline Onyx & & \\
$\quad$ Katsaridis et al. (2008) & & \\
$\quad$ Van Rooij et al. $(2007)^{18)}$ & $15.9 \%$ & $4.0 \%$ \\
NBCA & & \\
$\quad$ Debrun et al. (1997) ${ }^{19)}$ & $5.6 \%$ & $7.4 \%$ \\
$\quad$ Li et al. (2005) & $33 \%$ & $2.4 \%$ \\
Eudragit-E & & \\
$\quad$ Our study & $22.7 \%$ & $10.0 \%$ \\
\hline
\end{tabular}

NBCA: n-butyl 2-cyanoacrylate. 
the interaction between positively charged polymers and negatively charged blood cells. Chronic inflammatory reactions were, however, also observed in blood vessels of both humans and animals that were embolized with Onyx. ${ }^{24,25)}$ NBCA was also reported to cause mild-to-severe inflammatory reactions in both humans and animals. ${ }^{26-28)}$ Therefore, inflammation of embolized vessels is not unique to Eudragit-E.

Obstacles to the widespread use of Eudragit-E that are related to convenience may exist. Unfortunately, the 10\% Eudragit-E mixture is not supplied in ready-to-use vials. Use of the Eudragit-E mixture requires approval by an Institutional Review Board, and prior informed consent from patients and their family is also definitely required. Our use of Euragit-E was approved by our Institutional Review Board and accepted by patients and their family in all cases. We prepared 10\% Eudragit-E vials in our own pharmacy.

Onyx was approved for brain AVM embolization in 2009 in Japan, while Eudragit-E and NBCA were not at the time of submission of this article. However, we believe that the use of Eudragit-E is advantageous for some patients. The best indication of Eudragit-E is for cases with single fistula and low flow shunting. Good indication we believe is for cases with deep or eloquent location which may require subsequent stereotactic radiosurgery. We believe Eudragit-E is more, or at least equivalently, useful than Onyx for cases requiring subsequent radiosurgery or repeated embolization. Those AVMs with complex angiostructure that makes it difficult to produce Onyx plug may also be a good indication of Eudragit-E. Poor indication may be those with high flow shunting, but we believe that Eudragit-E can still be used if the proximal flow control technique is accompanied.

\section{Conclusion}

Eudragit-E is a non-adhesive, liquid embolic material that is easy to handle and is compatible with any type of microcatheter. Eudragit-E embolization is especially useful as a pretreatment for subsequent radiosurgery or surgery. The safety and effectiveness of Eudragit-E embolization were satisfactory.

\section{Conflicts of Interest Disclosure}

The authors have no personal, financial, or institutional conflicts of interest in any of the drugs, materials, or devices in the article. All authors who are members of The Japan Neurosurgical Society (JNS) have registered online Self-reported COI Disclosure Statement Forms through the website for JNS members.

\section{References}

1) Luessenhop AJ, Spence WT: Artificial embolization of cerebral arteries. Report of use in a case of arteriovenous malformation. J Am Med Assoc 172: 1153-1155, 1960

2) Matsumaru Y, Hyodo A, Nose T, Hirano T, Ohashi S: Embolic materials for endovascular treatment of cerebral lesions. J Biomater Sci Polym Ed 8: 555-569, 1997

3) Hyodo A, Nakai Y, Matsumaru Y, Kato N, Nose T, Harakuni T, Tsurushima H, Saito A, Yoshii Y: Endovascular treatment of intracranial AVM. Kor $J$ Cerebrovascular Disease 1: 82-87, 1999

4) Yamashita K, Taki W, Iwata H, Kikuchi H: A cationic polymer, Eudragit-E, as a new liquid embolic material for arteriovenous malformations. Neuroradiology 38(Suppl 1): S151-S156, 1996

5) Bogataj M, Mrhar A, Kristl A, Kozjek F: Eudragit E microspheres containing bacampicillin: preparation by solvent removal methods. J Microencapsul 8: 401-406, 1991

6) Lehmann K, Dreher D: The use of aqueous syntheticpolymer dispersions for coating pharmaceutical dosage forms. Drugs Made in Germany 19: 126-136, 1973

7) Oishi H, Yoshida K, Tange Y, Tsuji O, Sonobe M: Treatment of a scalp arteriovenous malformation by a combination of embolization and surgical removal. Interv Neuroradiol 8: 293-297, 2002

8) Yamamoto M, Akabane A, Matsumaru Y, Higuchi Y, Kasuya H, Urakawa Y: Long-term follow-up results of intentional 2-stage Gamma Knife surgery with an interval of at least 3 years for arteriovenous malformations larger than $10 \mathrm{~cm}^{3}$. J Neurosurg 117(Suppl): 126-134, 2012

9) Arakawa H, Murayama Y, Davis CR, Howard DL, Baumgardner WL, Marks MP, Do HM: Endovascular embolization of the swine rete mirabile with Eudragit-E 100 polymer. AJNR Am J Neuroradiol 28: 1191-1196, 2007

10) Loy DN, Rich KM, Simpson J, Dorward I, Santanam L, Derdeyn CP: Time-of-flight magnetic resonance angiography imaging of a residual arteriovenous malformation nidus after Onyx embolization for stereotactic radiosurgery planning. Technical note. Neurosurg Focus 26: E13: 1-4, 2009

11) Shtraus N, Schifter D, Corn BW, Maimon S, Alani S, Frolov V, Matceyevsky D, Kanner AA: Radiosurgical treatment planning of AVM following embolization with Onyx: possible dosage error in treatment planning can be averted. J Neurooncol 98: 271-276, 2010

12) Taylor CL, Dutton K, Rappard G, Pride GL, Replogle R, Purdy PD, White J, Giller C, Kopitnik TA, Samson DS: Complications of preoperative embolization of cerebral arteriovenous malformations. J Neurosurg 100: 810-812, 2004

13) Weber W, Kis B, Siekmann R, Jans P, Laumer R, Kühne D: Preoperative embolization of intrac- 
ranial arteriovenous malformations with Onyx. Neurosurgery 61: 244-252; discussion 252-254, 2007

14) Pierot L, Kadziolka K, Litré F, Rousseaux P: Combined treatment of brain AVMs with use of Onyx embolization followed by radiosurgery. AJNR Am J Neuroradiol 34: 1395-1400, 2013

15) Zabel-du Bois A, Milker-Zabel S, Huber P, Schlegel W, Debus J: Risk of hemorrhage and obliteration rates of LINAC-based radiosurgery for cerebral arteriovenous malformations treated after prior partial embolization. Int J Radiat Oncol Biol Phys 68: 999-1003, 2007

16) Panagiotopoulos V, Gizewski E, Asgari S, Regel J, Forsting M, Wanke I: Embolization of intracranial arteriovenous malformations with ethylene-vinyl alcohol copolymer (Onyx). AJNR Am J Neuroradiol 30: 99-106, 2009

17) Katsaridis V, Papagiannaki C, Aimar E: Curative embolization of cerebral arteriovenous malformations (AVMs) with Onyx in 101 patients. Neuroradiology 50: 589-597, 2008

18) van Rooij WJ, Sluzewski M, Beute GN: Brain AVM embolization with Onyx. AJNR Am J Neuroradiol 28: 172-177; discussion 178, 2007

19) Debrun GM, Aletich V, Ausman JI, Charbel F, Dujovny M: Embolization of the nidus of brain arteriovenous malformations with n-butyl cyanoacrylate. Neurosurgery 40: 112-120; discussion 120-121, 1997

20) Li TL, Fang B, He XY, Duan CZ, Wang QJ, Zhao QP, Huan QY: Complication analysis of 469 brain arteriovenous malformations treated with N-butyl cyanoacrylate. Interv Neuroradiol 11: 141-148, 2005

21) Andrade-Souza YM, Ramani M, Scora D, Tsao MN, terBrugge K, Schwartz ML: Embolization before radiosurgery reduces the obliteration rate of arteriovenous malformations. Neurosurgery 60: 443-451; discussion 451-452, 2007

22) Blackburn SL, Ashley WW, Rich KM, Simpson JR, Drzymala RE, Ray WZ, Moran CJ, Cross DT, Chicoine MR, Dacey RG, Derdeyn CP, Zipfel GJ:
Combined endovascular embolization and stereotactic radiosurgery in the treatment of large arteriovenous malformations. J Neurosurg 114: 1758-1767, 2011

23) Sampei K, Hashimoto N, Kazekawa K, Tsukahara T, Iwata $\mathrm{H}$, Takaichi S: Histological changes in brain tissue and vasculature after intracarotid infusion of organic solvents in rats. Neuroradiology 38: 291-294, 1996

24) Murayama Y, Viñuela F, Ulhoa A, Akiba Y, Duckwiler GR, Gobin YP, Vinters HV, Greff RJ: Nonadhesive liquid embolic agent for cerebral arteriovenous malformations: preliminary histopathological studies in swine rete mirabile. Neurosurgery 43: 1164-1175, 1998

25) Jahan R, Murayama Y, Gobin YP, Duckwiler GR, Vinters HV, Viñuela F: Embolization of arteriovenous malformations with Onyx: clinicopathological experience in 23 patients. Neurosurgery 48: 984-995; discussion 995-997, 2001

26) Brothers MF, Kaufmann JC, Fox AJ, Deveikis JP: n-Butyl 2-cyanoacrylate-substitute for IBCA in interventional neuroradiology: histopathologic and polymerization time studies. AJNR Am J Neuroradiol 10: 777-786, 1989

27) Duffner F, Ritz R, Bornemann A, Freudenstein D, Wiendl H, Siekmann R: Combined therapy of cerebral arteriovenous malformations: histological differences between a non-adhesive liquid embolic agent and n-butyl 2-cyanoacrylate (NBCA). Clin Neuropathol 21: 13-17, 2002

28) Gruber A, Mazal PR, Bavinzski G, Killer M, Budka H, Richling B: Repermeation of partially embolized cerebral arteriovenous malformations: a clinical, radiologic, and histologic study. AJNR Am J Neuroradiol 17: 1323-1331, 1996

Address reprint requests to: Goichiro Tamura, MD, Department of Neurosurgery, Mito Medical Center, National Health Organization, 280 Sakuranosato, Ibarakimachi, Higashi-ibaraki, Ibaraki 311-3193, Japan. e-mail: g-tamura@umin.ac.jp 